\title{
First-Principles Studies of Li Nucleation on Graphene
}

\author{
Mingjie Liu, ${ }^{1}$ Alex Kutana, ${ }^{1}$ Yuanyue Liu, ${ }^{1}$ and Boris I. Yakobson ${ }^{1,2,3, \#}$ \\ ${ }^{1}$ Department of Materials Science and Nanoengineering, ${ }^{2}$ Department of Chemistry, and ${ }^{3}$ Smalley \\ Institute for Nanoscale Science and Technology, Rice University, Houston, Texas 77005, USA \\ \#e-mail: biy@rice.edu
}

\begin{abstract}
:
We study the Li clustering process on graphene and obtain the geometry, nucleation barrier and electronic structure of the clusters using first-principles calculations. We estimate the concentration-dependent nucleation barrier for $\mathrm{Li}$ on graphene. While the nucleation occurs more readily with increasing $\mathrm{Li}$ concentration, possibly leading to the dendrite formation and failure of the Li-ion battery, the existence of the barrier delays nucleation and may allow Li storage on graphene. Our electronic structure and charge transfer analyses reveal how the fully-ionized $\mathrm{Li}$ adatoms transform to metallic $\mathrm{Li}$ during the cluster growth on graphene.
\end{abstract}

\section{TOC Graphic:}

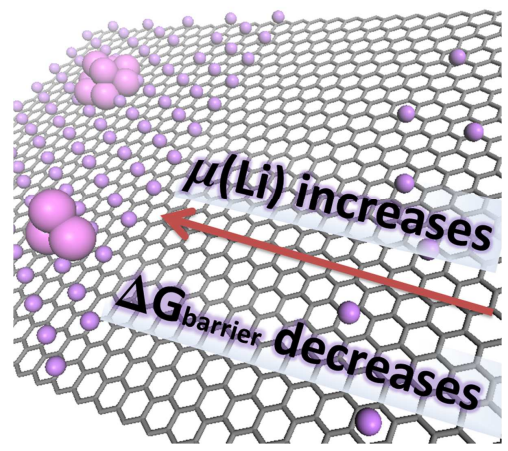

Key words: Li-ion battery, nucleation, cluster, graphene, first-principles

Graphite has been used as an anode material for Li-ion battery since the 1990s. The capacity of the Li-ion battery with the graphite anode is known to be $372 \mathrm{mAh} / \mathrm{g}$, corresponding to a $\mathrm{LiC}_{6}$ configuration, where intercalated Li ions are distributed on every other hexagon of the graphene lattice. ${ }^{1}$ Dendrite growth and $\mathrm{Li}$ atom plating are the main problems which limit the operation and can cause the failure of Li-ion batteries. ${ }^{2,3}$ Both problems are related to the Li cluster nucleation on graphite. Recently, other carbon materials such as graphene ${ }^{4,5,6}$ and carbon nanotubes $(\mathrm{CNTs})^{7,8}$ have been proposed as replacement anode materials. Both materials have an added advantage of accessible large surface area, which could potentially increase the capacity of the battery. Although the thermal capacity of graphene anode has since been demonstrated to be lower than that of graphite in both experiments and theoretical studies, $,{ }^{9}, 10$ the nucleation of $\mathrm{Li}$ on graphene has barely been studied. ${ }^{11,12}$ The dendrite formation has been explored and simulated from the macroscopic point of view, ${ }^{2}$ where it was assumed that a nucleation center has been formed already. ${ }^{13}$ Investigating the conditions under which a nucleation center forms requires atomistic simulation and modeling. It is known that metal atom clustering happens in carbon materials, 
such as on SWNT. ${ }^{14}$ In this work, we applied first-principles calculations to study Li clusters on graphene. Our results show that although the binding energy of $\mathrm{Li}$ on graphene is weaker than $\mathrm{Li}$ in the bulk, the nucleation barrier may prevent the phase separation of Li clusters and graphene. The nucleation barrier depends on the chemical potential of $\mathrm{Li}$ which in turn depends on the concentration of $\mathrm{Li}$ on graphene. We applied first-principles calculations and macroscopic modeling to roughly estimate the nucleation size and energy barrier under various Li concentrations. We also analyzed the charge transfer during nucleation, gaining insights into the interactions between the Li ions and graphene as well as the nucleation process.

We applied density functional theory (DFT) with generalized gradient approximation (GGA) as implemented in VASP to relax the atomic structures and obtain the total energies. The projector augmented waves (PAW) and PW91 functional have been used. ${ }^{15,16}$ The plane wave energy cutoff was $400 \mathrm{eV}$. The sequence of Li concentrations on graphene was calculated by increasing the size of the graphene supercell from $5 \times 5$, to $6 \times 6$, to $7 \times 7$, to $8 \times 8$, to $9 \times 9$. For each supercell, the convergence of Kpoint mesh was tested. In cluster calculations, a $9 \times 9$ graphene supercell was used with a vacuum space of $15 \AA$ along the $\mathrm{z}$ direction. The Brillouin zone was sampled using a $\Gamma$-centered $3 \times 3 \times 1 \mathrm{~K}$-point grid in all cases. For electronic structure calculations, a $6 \times 6$ graphene supercell was used. Since the bonding between $\mathrm{Li}$ and graphene is mostly ionic, ${ }^{17}$ the role of van der Waals interactions is minor, and van der Waals corrections are not essential here. We also note that in systems with well-pronounced charge transfer states such as ours, semilocal functionals may not yield accurate results due to their well-known delocalization error. To verify the accuracy of PW91 functional, we performed an additional calculation using a hybrid HSE functional for the adsorption energy of $\mathrm{LiC}_{6}$. We obtained values of -0.95 and -0.92 eV/atom for the Li adsorption energy with PW91 and HSE functionals, respectively. As expected, the interaction energy given by HSE is smaller, consistent with the fact that (semi)local functionals overestimate binding of charge transfer states. However, the difference between the two values is not very large, validating applicability of PW91 to this system.

The structure of Li clusters on graphene. We considered clusters with the numbers of atoms $n$ up to 13; Fig. 1 shows the obtained low-energy configurations for clusters with $n$ up to 11. Unlike graphite, the adsorption energy of a single $\mathrm{Li}$ atom on graphene is higher than the bulk $\mathrm{Li}$; therefore, in the thermodynamical limit, $\mathrm{Li}$ atoms would tend to aggregate into large clusters rather than adsorb on graphene separately. ${ }^{9,10}$ However, there will be a nucleation barrier due to the high surface energy of small clusters. We searched for the lowest energy structures by considering several possible configurations for each cluster. For smaller clusters $(n=3,4,5)$, all possible configurations were searched, and clusters with the lowest energy were selected as the ground state. For larger clusters $(n>5)$, global optimization was impractical, and two alternative approaches were used to identify low energy structures. One is to simply add an atom to a previously found low energy (n-1)-atom cluster. Another is to start with the ground state geometry in vacuum, ${ }^{18,19}$ bring the cluster in contact with graphene, and perform relaxation. In some cases $(n=6,8,9)$, both approaches led to same geometries. For the remaining clusters $(n=7,10,11,12,13)$, the first method yielded geometries with the energy that is lower than that obtained by starting from the equilibrium geometry in vacuum. It is possible that the found geometries do not represent the true ground states for these clusters; however it is likely that their energies are quite close to them, considering the inherent polymorphism of few-atom metal clusters. 


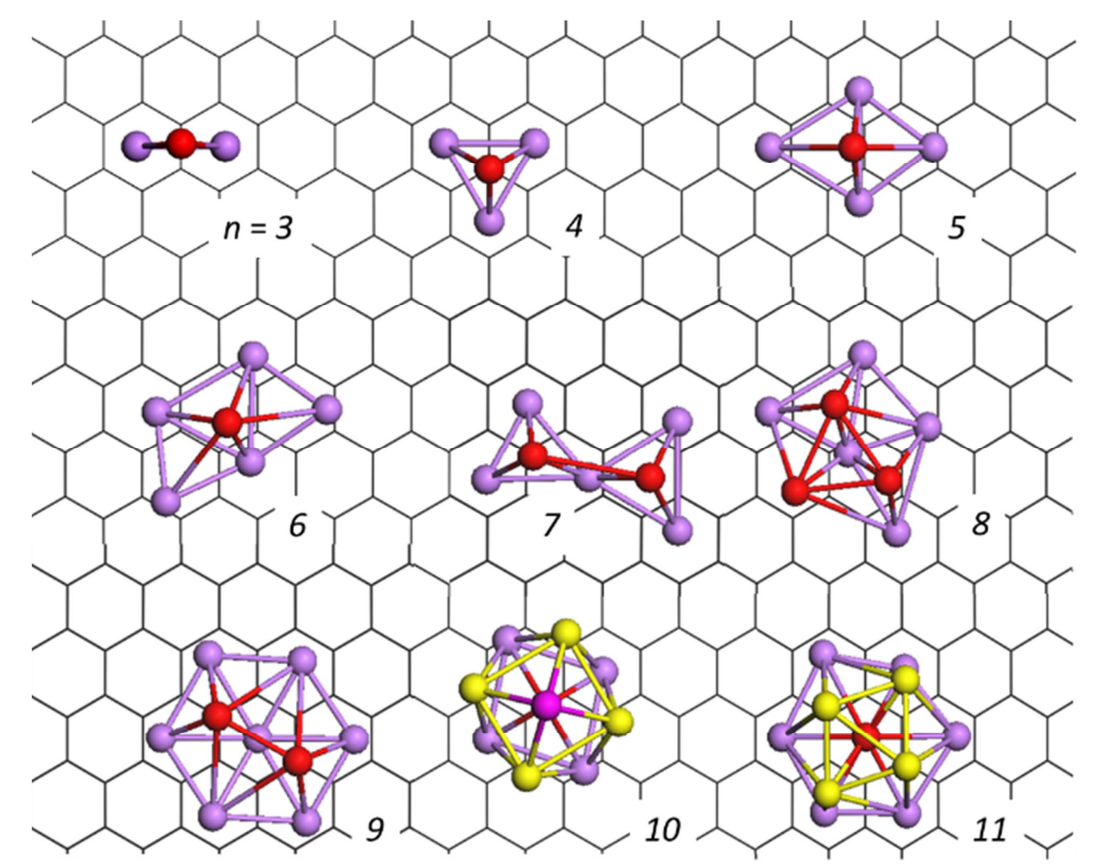

Figure 1. The most stable structures of Li clusters on graphene from $\mathrm{n}=3$ to 11 . Balls represent $\mathrm{Li}$ atoms and are colored in the order of increasing distance from graphene: purple (closest to graphene), red, yellow, and light purple (farthest from graphene).

The most stable configurations of $\mathrm{Li}$ clusters $\left(\mathrm{Li}_{\mathrm{n}}\right)$ on graphene are not necessary the same as in vacuum. For $n=2$, the $\mathrm{Li}$ atoms form a dimer with the optimized distance of 2.65-2.78 $\AA$ in vacuum; ${ }^{20}$ however, for two $\mathrm{Li}$ atoms attached to graphene, the effective pair interaction is repulsive, and the formation energy of the dimer is larger than the sum of bonding energies of individual Li atoms. Hence, the dimer structure for $\mathrm{Li}_{2}$ is not favorable on graphene. For $n=3, \mathrm{Li}$ atoms form an isosceles triangle in vacuum. ${ }^{18} \mathrm{The}^{\mathrm{Li}} \mathrm{i}_{3}$ on graphene forms isosceles triangle as well but the third atom prefers to stay above the other two rather than attaching on graphene to form the equilateral triangle. It points to the start of nucleation, where the cluster prefers forming a three-dimensional shape rather than being attached to graphene surface. For $n=4$, the $\mathrm{Li}$ atoms form a rhombus in vacuum, but the most stable structure for $\mathrm{Li}_{4}$ on graphene is a tetrahedron. ${ }^{18}$ All other cluster configurations for $n=2-5$ and their energy differences are listed in the Supporting Information. The observed non-intuitive behavior for the small Li clusters $(n<7)$ to prefer planar configurations in vacuum, ${ }^{18}$ but form three-dimensional shapes on graphene is discussed below from the electronic structure point of view.

The cluster formation energy and macroscopic model. Based on the determined most stable structures, we estimate the formation energy of clusters. The formation free energy $\Delta \mathrm{G}(n)$ of each cluster at concentration $x$ is defined as

$$
\Delta \mathrm{G}(n) \equiv E\left(\mathrm{Li}_{\mathrm{n}} @ \text { graphene }\right)-E(\text { graphene })-n \cdot \mu\left(\mathrm{Li}_{\mathrm{Li}}^{\mathrm{x}} \mathrm{C}\right)
$$

where $\mu\left(\mathrm{Li}_{\mathrm{Li}}^{\mathrm{x}} \mathrm{C}\right)$ is the chemical potential of $\mathrm{Li}$ under various conditions. In $\mathrm{Li}$-ion batteries, the $\mathrm{Li}^{+}$ ions arrive at the surface and are adsorbed on graphene first. ${ }^{21} \mathrm{We}$ use this adsorbed phase as a reference, and define the chemical potential of $\mathrm{Li}$ as 


$$
\mu\left(\mathrm{Li}_{\mathrm{Li}_{x} \mathrm{C}}\right)=\left[E\left(\mathrm{Li}_{x} \mathrm{C}\right)-E(\mathrm{C})\right] / x-T S(x)
$$

The nucleation process depends strongly on the chemical potential of $\mathrm{Li}$ atoms. For instance, if the chemical potential is chosen to be that of atomic $\mathrm{Li}$, the cluster energy would be negative, and the formation of Li clusters is strongly favored, whereas if the chemical potential is chosen to be that of $\mathrm{Li}$ in the bulk phase, the formation energy is positive, meaning that cluster formation is unlikely. When the adsorbed phase is used as a reference, the chemical potential of $\mathrm{Li}$ will be concentration-dependent. We compute the $\mu\left(\mathrm{Li} @ \mathrm{Li}_{\mathrm{x}} \mathrm{C}\right)$ for $\mathrm{Li}: \mathrm{C}$ ratios ranging from 1:162 to 1:6. We also include the configurational entropy correction at a dilute limit into the chemical potential of the reference phase. The configurational entropy of the clusters is much smaller than that of the reference phase and can be neglected. The entropy of the reference phase is estimated by counting the number of adsorption configuration at a given concentration. The entropy $S$ (per adsorbed Li atom) of the adsorbed phase is given by:

$$
-S(x)=k_{B}[\ln 2 x+(1-2 x) \ln (1-2 x) / 2 x]
$$

Here, $x=L i: C$, and $k_{B}$ is the Boltzmann constant. ${ }^{22}$ The temperature was taken to be $300 \mathrm{~K}$.

We also estimate the formation energy of Li clusters on graphene using a macroscopic model. The model is useful when considering cluster sizes that are not accessible with the atomistic calculations. The spherical model of Li clusters can be used to do a quick estimation but a more accurate equilibrium shape of a Li cluster in vacuum should be determined utilizing Wulff construction. ${ }^{23}$ The equilibrium shape of $\mathrm{Li}$ clusters in vacuum is obtained by minimizing the surface energy of cluster $E_{s}$ at a given size. If the cluster shape is a polyhedron, the formation energy with respect to the bulk phase is given by:

$$
E_{s}=\sum_{i} \gamma_{i} A_{i}
$$

where $\gamma_{i}$ is the surface energy of the $i$ th facet of the polyhedron and $A_{i}$ is the area of the facet. According to the Wulff theorem, the distances of the crystal facets from the center are proportional to the facet surface energies when the crystal is at equilibrium. Based on cluster size $n$, one can estimate the volume of Li clusters from the atomic volume, and by simple geometry analysis according to Fig.2b, the change of the surface area $A_{i}$ with $n$ can be derived. We construct the equilibrium shape using the values of 29 $\mathrm{meV} / \AA^{2}, 31 \mathrm{meV} / \AA^{2}$, and $35 \mathrm{meV} / \AA^{2}$, for the formation energies of the (100), (110), and (111) facets of $\mathrm{Li}$, respectively, as obtained with the DFT calculation using GGA. ${ }^{24}$ The equilibrium particle shape is shown in Fig.2b. After accounting for various contributions, the particle formation free energy $\Delta G(n)$ is given by:

$$
\Delta G(n)=E_{s}+E_{\text {bulk }}-A_{(100)} \gamma_{\text {interface }}-\mu n
$$

Here, $n$ is the number of atoms in the cluster, $E_{b u l k}$ is the atomization energy of bulk $\mathrm{Li}, A_{(100)}$ is the area of the particle (100) facet, and $\mu$ is the chemical potential of the reference phase (in this case, a submonolayer amount of $\mathrm{Li}$ on graphene at a given concentration). We chose the (100) facet because it has the largest surface area. For the interface energy $\gamma_{\text {interface }}$ of $\operatorname{Li}(100)$ on graphene, we obtained a value of $14 \mathrm{meV} / \AA^{2}$, by calculating the graphene-Li interaction energy for a Li slab on graphene with six Li layers. The cluster formation curves based on the macroscopic cluster model are shown in dashed lines in Fig.2a. The few-atoms Li clusters considered in our work do not have facets corresponding to well- 
defined crystal surfaces that normally exist in large metal particles. Hence, the model of a bulk crystal truncated by several low-energy crystalline planes is not directly applicable in this case. Not surprisingly, the model, when used in an unmodified form, predicts formation energies that are different (too high) from the actual cluster formation energies. We also considered an altered model assuming the same scaling of the formation energy, $E \sim n^{2 / 3}$, but with a prefactor that is different in case of very small clusters. The prefactor was obtained by fitting the calculated formation energies, and can be conveniently incorporated into the interface energy. We got the value of $\sim 150 \mathrm{meV} / \AA^{2}$ for the effective interface energy, with the fitting curves shown as solid lines in Fig.2a. It should be noticed that when the clusters become larger and approach the macroscopic limit, the nucleation behavior should be represented by the dashed lines. Any changes in the cluster shape upon the adsorption were neglected, and the formation energy on graphene was calculated using the equilibrium shape in vacuum.
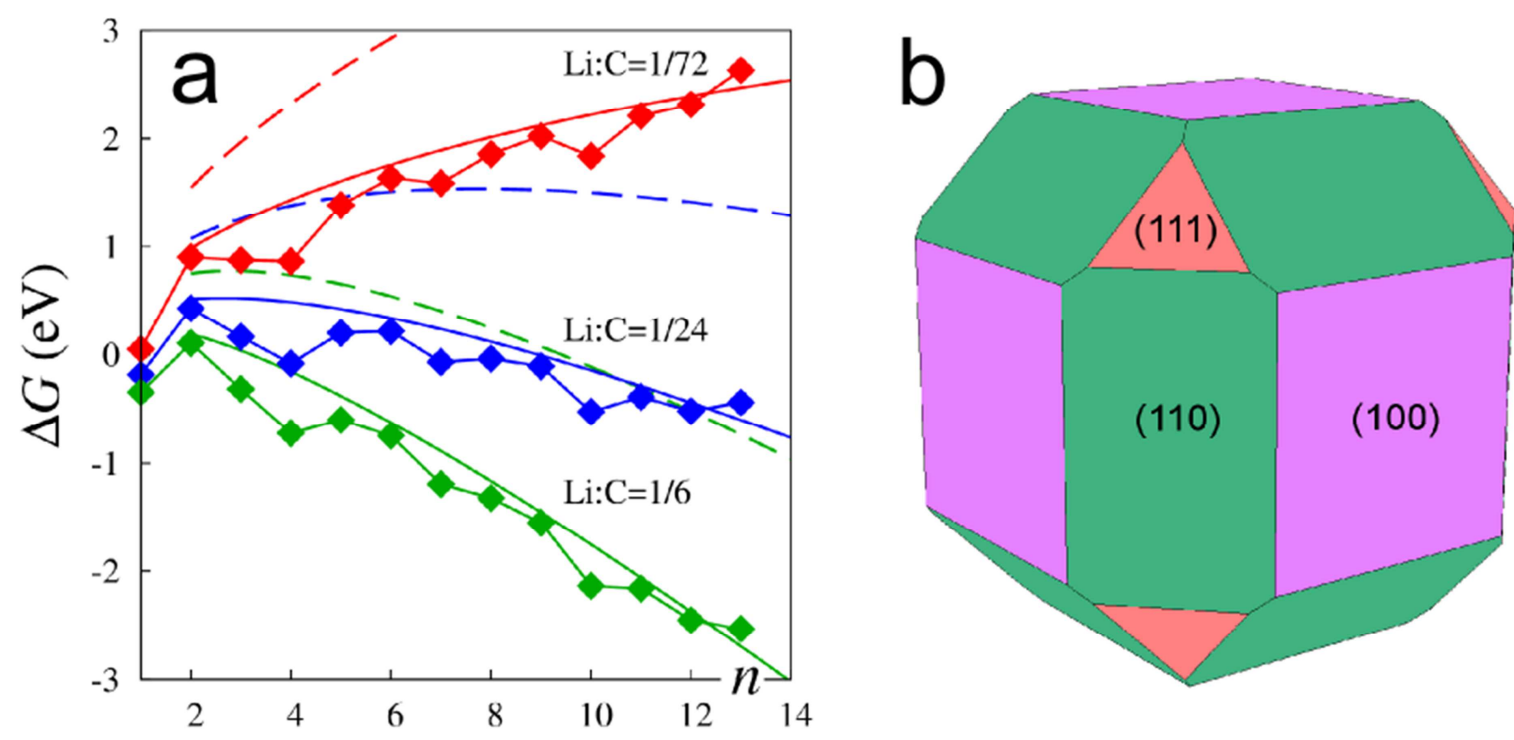

Figure 2. (a) Cluster formation energies at different chemical potentials: symbols represent atomistic results, as given by Eq.1; the dashed lines are obtained from the macroscopic Wulff construction model

(Eq.5), and solid lines show the fitting of Eq.5 by modifying the interface energy $\gamma_{\text {interface }}$. The equilibrium crystal shape from the Wulff construction is shown in (b).

The comparison of the cluster formation energy as predicted by Eq.5 and calculated formation energies of actual clusters by Eq. 1 are shown in Fig.2a, with good agreement. Using the analytical expression, we get the critical cluster size and the nucleation barrier. The critical cluster sizes and nucleation barriers at various reference $\mathrm{Li}$ concentrations are listed in Table I. As we can see, the cluster sizes for nucleation are small, but the nucleation barriers are not negligible. For example, the smallest nucleation barrier at high concentration limit is around $0.27 \mathrm{eV}$; for the dilute case ( $\mathrm{Li}: \mathrm{C}=1: 162$ ), the barrier is as high as $\sim 14.7 \mathrm{eV}$. We conjecture that overcoming this nucleation barrier and forming a metal particle may be important for the initial nucleation of a dendrite. However, answering the question of how the dendrite that was nucleated by a Li nucleus proceeds to grow into mesoscopic and macroscopic sizes is beyond the scope of this work. 
Table I. Cluster sizes and the nucleation barriers obtained from the analytical cluster model at various reference Li concentrations on graphene.

\begin{tabular}{c|c|c}
\hline$L i: C$ & $N$ & $\Delta G_{\text {barrier }}(\mathrm{eV})$ \\
\hline $1: 6$ & 1 & 0.27 \\
$1: 24$ & 3 & 0.53 \\
$1: 72$ & 40 & 3.23 \\
\hline
\end{tabular}

The electronic structure and electron transfer during clustering. Based on the previous discussions, the interaction between $\mathrm{Li}$ clusters and graphene is stronger than that of neutral metallic bulk Li on graphene, which indicates the stronger ionic bonding and charge transfer from Li to graphene. A single Li adatom binds to graphene by donating its electron to the delocalized $\pi$ states of graphene, and becomes fully ionized. ${ }^{25}$ When the cluster is formed, the Li electrons should be localized inside the cluster, similar to bulk $\mathrm{Li}$ where each $\mathrm{Li}$ has zero net charge. At intermediate cluster sizes, the transition from the adatom to bulk behavior occurs. For $\mathrm{Li}_{2}$ in vacuum, the $\mathrm{Li}$ electrons pair up and form the $\mathrm{Li}-\mathrm{Li}$ bond. However, when adsorbed on graphene, our calculations indicate that the Li electrons are mainly transferred to graphene by filling up the upper Dirac cone, as shown in Fig.3a. It suggests that the $\mathrm{Li}_{2}$ is ionized and each $\mathrm{Li}$ carries +1 charge. For $\mathrm{Li}_{3}$, a flat band appears below the Fermi level. Its charge density distribution at $\Gamma$ point indicates that two electrons are shared between the $\mathrm{Li}_{3}$ cluster (Fig.3b). The remaining electron is transferred to graphene. Therefore, on average, each $\mathrm{Li}$ carries $+1 / 3$ charge. For $\mathrm{Li}_{4}$ (Fig.3c), two electrons are localized inside the cluster, and thus each $\mathrm{Li}$ has $+1 / 2$ charge averagely. As the cluster grows larger, the net charge of $\mathrm{Li}$ asymptotically approaches the zero charge of bulk Li. The low energy of the electron localized inside the Li clusters (from $\mathrm{Li}_{3}$ to $\mathrm{Li}_{4}$ ) might be an additional driving force for the nucleation. To test the extent of applicability of DFT band structures to describe quasiparticle energies in real systems, we have additionally carried out a GW calculation for the band structure of $\mathrm{LiC}_{6}$. The plot comparing the PW91 and GW band structures of $\mathrm{LiC}_{6}$ is shown in Fig.S3. As can be seen from the figure, the main impact of including many-body effects into the quasiparticle energies is the lowering of quasiparticle bands with respect to the Fermi level. Another observation is that the electron occupation of the $\mathrm{Li}$ band is higher in $\mathrm{GW}$, suggesting decreased charge transfer from $\mathrm{Li}$ to graphene relative to PW91 results. From the above comparison, we expect that our qualitative analysis of the charge transfer process from adsorbed $\mathrm{Li}$ to graphene should remain valid. 


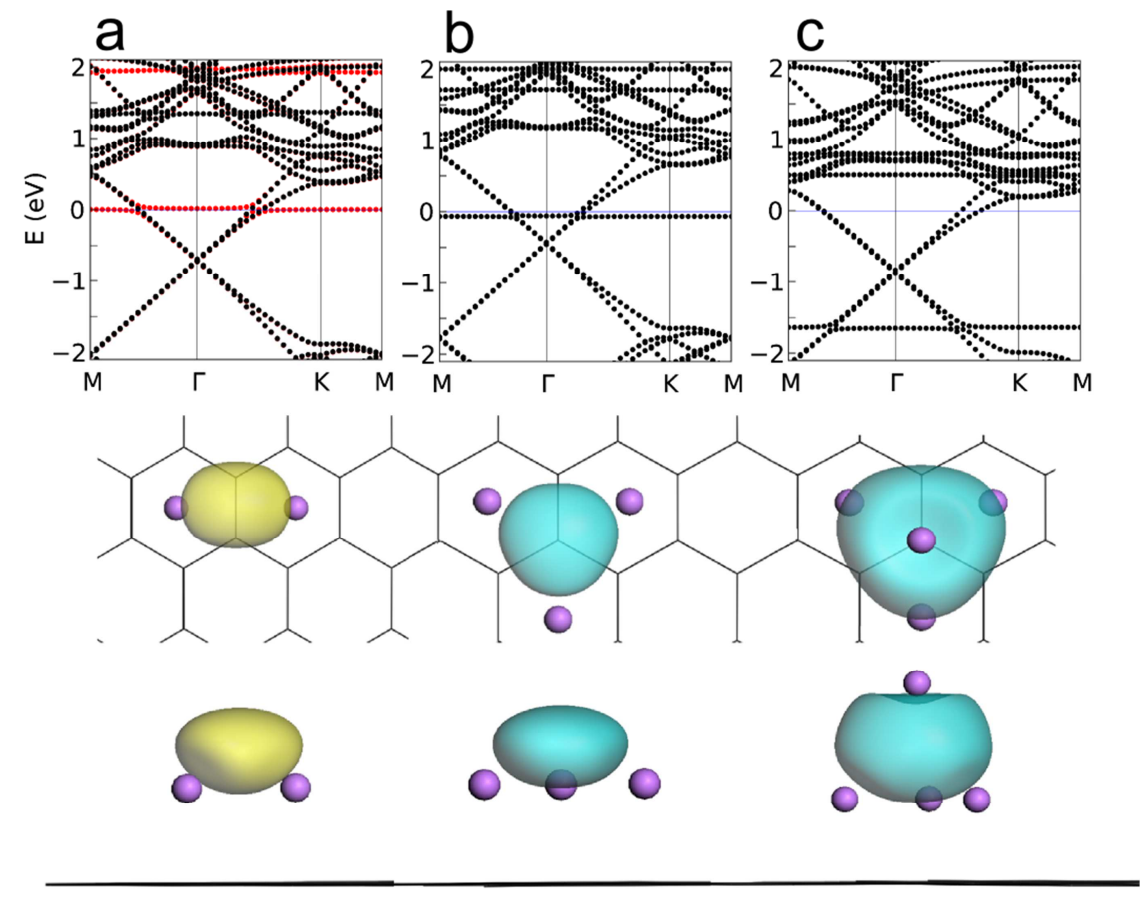

Figure 3. Electronic structure of Li cluster on graphene for (a) $\mathrm{Li}_{2}$ (b) $\mathrm{Li}_{3}$ (c) $\mathrm{Li}_{4}$. Red and black dots in (a) show the spin-polarized states of the magnetic $\mathrm{Li}_{2}$. The $\mathrm{Li}_{3}$ and $\mathrm{Li}_{4}$ are non-magnetic and thus the states are doubly-occupied. The Fermi level is set to zero. The charge density distribution of the flat band at $\Gamma$ point is shown in the bottom panel. For (a), the isosurface is displayed in yellow to indicate the state is unoccupied.

In summary, we investigated the Li cluster formation process using first-principles calculations and found that the Li nucleation barrier depends strongly on the concentration of $\mathrm{Li}$ ions on graphene. This nucleation barrier would prevent the phase separation from occurring under certain concentrations. The electronic structure of those clusters on graphene indicates that the low energy electrons localized inside the Li clusters might be the driving force for the nucleation.

\section{Acknowledgment:}

We thank X. Zou for fruitful discussions and assistance in GW calculations. The work is supported by the Honda Research Institute USA. The computational resources were provided by DAVinCI funded by the NSF.

Supporting Information Available: Calculations of the interaction energy of two Li atoms on graphene as a function of their separation, relative energies of various configurations of Li clusters on graphene with $n=3-5$, and $\mathrm{GW}$ band structure of $\mathrm{LiC}_{6}$. This material is available free of charge via the Internet at http://pubs.acs.org

\section{References:}


(1) Goodenough, J. B.; Park, K.-S. The Li-Ion Rechargeable Battery: A Perspective. J. Am. Chem. Soc. 2013, 135, 1167-1176.

(2) Harris, S. J.; Timmons, A.; Baker, D. R.; Monroe, C. Direct in situ Measurements of Li Transport in Li-Ion Battery Negative Electrodes. Chem. Phys. Lett. 2010, 485, 265-274.

(3) Tarascon, J.-M.; Armand, M. Issues and Challenges Facing Rechargeable Lithium Batteries. Nature 2001, 414, 359-367.

(4) Yoo, E.; Kim, J.; Hosono, E.; Zhou, H.-s.; Kudo, T.; Honma, I. Large Reversible Li Storage of Graphene Nanosheet Families for Use in Rechargeable Lithium Ion Batteries. Nano Lett. 2008, 8, 22772282.

(5) Wang, G.; Shen, X.; Yao, J.; Park, J. Graphene Nanosheets for Enhanced Lithium Storage in Lithium Ion Batteries. Carbon 2009, 47, 2049-2053.

(6) Lian, P.; Zhu, X.; Liang, S.; Li, Z.; Yang, W.; Wang, H. Large Reversible Capacity of High Quality Graphene Sheets as an Anode Material for Lithium-Ion Batteries. Electrochim. Acta 2010, 55, 3909-3914.

(7) Lee, S. W.; Yabuuchi, N.; Gallant, B. M.; Chen, S.; Kim, B.-S.; Hammond, P. T.; Shao-Horn, Y. High-power Lithium Batteries from Functionalized Carbon-Nanotube Electrodes. Nat. Nanotechnol. 2010, 5, 531-537.

(8) Kaskhedikar, N. A.; Maier, J. Lithium Storage in Carbon Nanostructures. Adv. Mater. 2009, 21, 2664-2680.

(9) Lee, E.; Persson, K. A. Li Absorption and Intercalation in Single Layer Graphene and Few Layer Graphene by First Principles. Nano Lett. 2012, 12, 4624-4628.

(10) Liu, Y.; Artyukhov, V. I.; Liu, M.; Harutyunyan, A. R.; Yakobson, B. I. Feasibility of Lithium Storage on Graphene and Its Derivatives. J. Phys. Chem. Lett. 2013, 4, 1737-1742.

(11) Fan, X.; Zheng, W.; Kuo, J.-L.; Singh, D. J. Adsorption of Single Li and the Formation of Small Li Clusters on Graphene for the Anode of Lithium-ion Batteries. ACS Appl. Mater. Interfaces 2013, 5, 7793-7797.

(12) Garay-Tapia, A.; Romero, A. H.; Barone, V. Lithium Adsorption on Graphene: From Isolated Adatoms to Metallic Sheets. J. Chem. Theory Comput. 2012, 8, 1064-1071.

(13) Mayers, M. Z.; Kaminski, J. W.; Miller III, T. F. Suppression of Dendrite Formation via Pulse Charging in Rechargeable Lithium Metal Batteries. J. Phys. Chem. C 2012, 116, 26214-26221.

(14) Krasnov, P. O.; Ding, F.; Singh, A. K.; Yakobson, B. I. Clustering of Sc on SWNT and Reduction of Hydrogen Uptake: Ab-Initio All-Electron Calculations. J. Phys. Chem. C 2007, 111, 1797717980.

(15) Perdew, J. P.; Chevary, J. A.; Vosko, S. H.; Jackson, K. A.; Pederson, M. R.; Singh, D. J.; Fiolhais, C. Atoms, Molecules, Solids, and Surfaces: Applications of the Generalized Gradient Approximation for Exchange and Correlation. Phys. Rev. B 1992, 46, 6671.

(16) Perdew, J. P.; Chevary, J. A.; Vosko, S. H.; Jackson, K. A.; Pederson, M. R.; Singh, D. J.; Fiolhais, C. Erratum: Atoms, Molecules, Solids, and Surfaces: Applications of the Generalized Gradient Approximation for Exchange and Correlation. Phys. Rev. B 1993, 48, 4978.

(17) Chan, K. T.; Neaton, J. B.; Cohen, M. L. First-Principles Study of Metal Adatom Adsorption on Graphene. Phys. Rev. B 2008, 77, 235430.

(18) Boustani, I.; Pewestorf, W.; Fantucci, P.; Bonaić-Koutecký, V.; Koutecký, J. Systematic Ab Initio Configuration-Interaction Study of Alkali-Metal Clusters: Relation between Electronic Structure and Geometry of Small Li Clusters. Phys. Rev. B 1987, 35, 9437.

(19) Fournier, R.; Cheng, J. B. Y.; Wong, A. Theoretical Study of the Structure of Lithium Clusters. J. Chem. Phys. 2003, 119, 9444-9454.

(20) Gardet, G.; Rogemond, F.; Chermette, H. Density Functional Theory Study of Some Structural and Energetic Properties of Small Lithium Clusters. J. Chem. Phys. 1996, 105, 9933-9947.

(21) Mandeltort, L.; Yates, J. T. Rapid Atomic Li Surface Diffusion and Intercalation on Graphite: A Surface Science Study. J. Phys. Chem. C 2012, 116, 24962-24967. 
(22) Landau, L. D., Statistical Physics, Third Edition, Part1: Volume 5 (Course of Theoretical Physics, Volume 5). Butterworth-Heinemann: Burlington, 1975.

(23) Herring, C. Some Theorems on the Free Energies of Crystal Surfaces. Phys. Rev. 1951, 82, 87-93.

(24) Doll, K.; Harrison, N.; Saunders, V. A Density Functional Study of Lithium Bulk and Surfaces. J. Phys-Condens. Mat. 1999, 11, 5007.

(25) Liu, Y.; Wang, Y. M.; Yakobson, B. I.; Wood, B. C. Origins of Lithium Binding in CarbonBased Lithium-Ion Battery Anodes. arXiv: 1401.66712014. 